\title{
e-Phaïstos
}

e-Phaïstos

Revue d'histoire des techniques / Journal of the history

of technology

IX-1 | 2021

Autour de Léonard de Vinci

\section{Le cheval de Léonard}

Réflexions sur la mise en image de l'art équestre à l'époque moderne

The Spirit of Leonardo's Horse in the Modern Equestrian Art

\section{Anne-Sophie Rieth}

\section{(2) OpenEdition}

\section{Journals}

Édition électronique

URL : https://journals.openedition.org/ephaistos/8933

DOI : 10.4000/ephaistos.8933

ISSN : 2552-0741

Éditeur

IHMC - Institut d'histoire moderne et contemporaine (UMR 8066)

Référence électronique

Anne-Sophie Rieth, « Le cheval de Léonard », e-Phaïstos [En ligne], IX-1 | 2021, mis en ligne le 27 avril 2021, consulté le 16 septembre 2021. URL : http://journals.openedition.org/ephaistos/8933 ; DOI : https://doi.org/10.4000/ephaistos.8933

Ce document a été généré automatiquement le 16 septembre 2021.

Tous droits réservés 


\title{
Le cheval de Léonard
}

\author{
Réflexions sur la mise en image de l'art équestre à l'époque moderne \\ The Spirit of Leonardo's Horse in the Modern Equestrian Art
}

Anne-Sophie Rieth

1 La place du cheval est majeure dans l'ensemble des réalités figuratives comme dans l'art. Cette "représentation des équidés est étroitement dépendante des intentions esthétiques d'un moment, d'un lieu, des variations contemporaines des canons artistiques dominants ", souligne Daniel Roche (Roche 2015 : 359). Léonard de Vinci (1452-1519) ne fait pas exception. Artiste à la recherche constante de la dynamique des corps de l'Homme en mouvement, il retranscrit avec génie ses expériences fonctionnelles et ses recherches anatomiques dans ses études graphiques. "Si son œuvre anatomique sert sa peinture, directement ou non, c'est davantage pour traduire son obsession de la dynamique, du jeu des formes, de la construction sans faille d'une œuvre où absolument aucun détail n'est laissé au hasard que par un désir réel d'appliquer à cet art les données concrètes de ses propres travaux ", précise Dominique Le Nen (Le Nen 2007 : 24). En fait, la conception artistique de Léonard fait écho aux questions portant sur les illustrations des traités techniques équestres et plus spécifiquement sur la représentation du geste technique, sur la façon de traduire sur une surface plane en deux dimensions, l'éphémère du geste et la sensibilité du ressenti. On y retrouve ce qu'Eugenio Garin appelle « l'art-science », qui consiste à comprendre comment la science impacte l'imitation du réel et de la nature (Garin 2002).

2 Allant dans ce sens, plusieurs artistes de la Renaissance ont réfléchi sur la forme à donner à cette représentation. On peut citer à titre d'exemple l'Equile Joannis Austriaci, traité rédigé par Jan Van der Straet (1523-1605) ou bien encore les planches des Chevaux de différents pays d'Antonio Tempesta (1555-1630). Entre réalité et idéal, l'enjeu est de se mettre au service du cavalier. Avec la multiplication des ouvrages naturalistes et le développement de l'imprimerie au XVI siècle, l'image a changé de statut. En effet, de simple élément de décoration, elle est devenue instrument du discours, outil de communication. Elle s'est donc installée dans la rhétorique des penseurs de la technique équestre, qui l'utilisent pour aider le lecteur à se construire une représentation du mouvement demandé et du geste pour l'obtenir. 
3 Dans la continuité du travail d'Armelle Fémelat sur les portraits équestres italiens des Trecento et Quattrocento et de l'exposition Leonardo da Vinci: A Life in Drawing ${ }^{1}$ qui a présenté une analyse fine des plus grands dessins du maitre, cet article se propose de mener une réflexion sur la conception d'outils d'analyse historique pour interroger la capture par l'écrit de l'immédiateté et de l'intentionnalité du geste équestre. Aussi, suivant l'esprit du travail de Léonard de Vinci, il s'agira d'analyser les représentations du cheval dans les traités techniques d'équitation des $\mathrm{XVII}^{\mathrm{e}}$ et $\mathrm{XVIII}{ }^{\mathrm{e}}$ siècles. Pour ce faire, nous interrogerons dans un premier temps la place du réalisme dans la représentation du geste du cheval et de celui du cavalier, en nous intéressant à l'équilibre entre la permanence du mouvement théorisé et la spécificité individuelle de l'instant perceptible à travers la réalité du sensible. Cela servira de contre-point à une seconde partie qui se focalisera sur la vision idéalisée tirée des justes proportions du réel. Enfin, dans une troisième partie, nous nous concentrerons sur la représentation équestre comme instrument pour imposer et transmettre les principes, les croyances et les valeurs politiques et sociales des grands de ce monde.

\section{Un modèle vivant}

4 L'art italien du XV siècle connaît une diffusion de concepts, de procédés techniques et d'innovations qui vont révolutionner les modalités de représentation du mouvement à travers le relief, les volumes, les perspectives, la notion de proportion. Apparenté au réalisme, ce mouvement aboutit à une transformation des corps à la recherche de la perfection d'une esthétique de la vérité corporelle, caractéristique de la Renaissance. La recherche de la compréhension du fonctionnement du corps de l'Homme pousse la guilde des peintres vers la dissection des cadavres et une collaboration avec les anatomistes. Au premier plan de cette tendance, on retrouve Michel-Ange (1475-1564), Raphaël (1483-1520), Andrea Del Sarto (1486-1530), et bien sûr Léonard de Vinci. 
Fig.1. Esquisse du cheval cabré

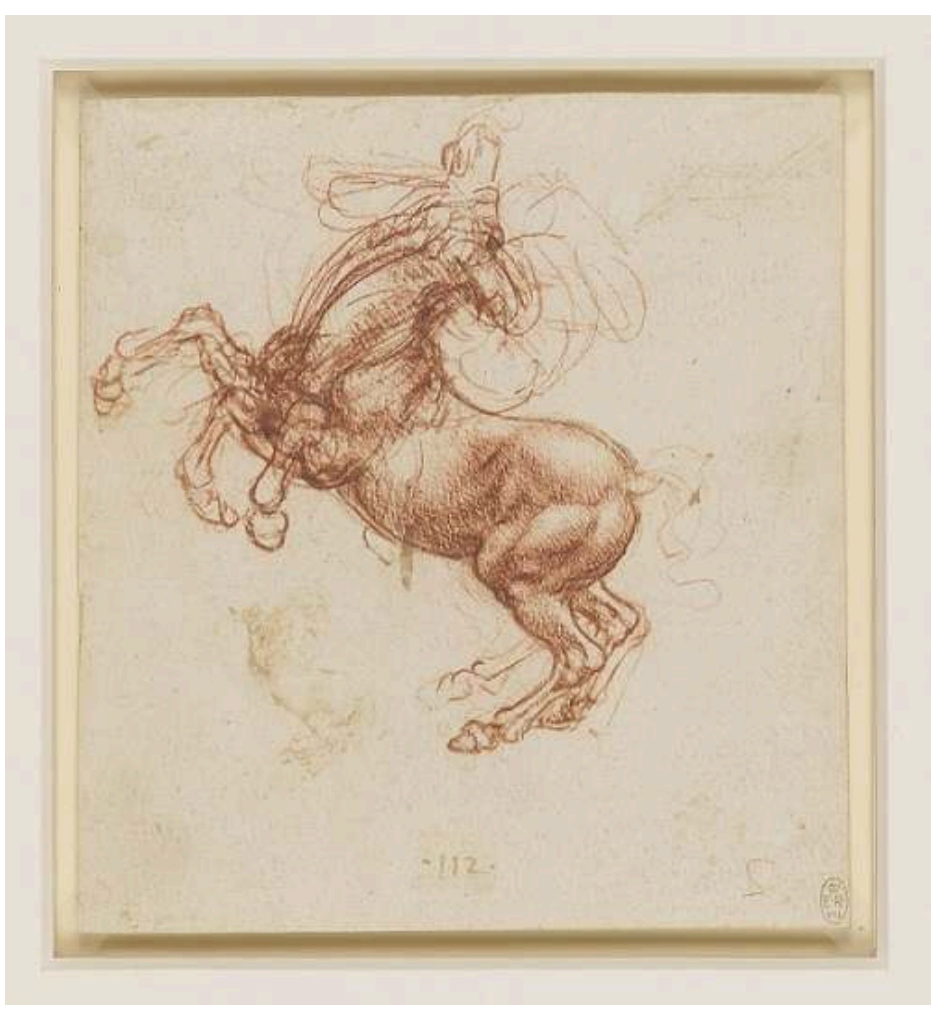

Dans cette étude graphique, Léonard de Vinci associe la mécanique du corps animal avec le dynamisme de l'éphémère du mouvement d'une encolure ou bien encore du posé d'un membre.

"A rearing horse c.1503-4. Redchalk, pen and ink | 15.3 x 14.2 cm (sheet of paper) | RCIN 912336 », Royal Collection Trust/@ Her Majesty Queen Elizabeth II 2021

5 À la recherche constante de la dynamique des corps de l'Homme en mouvement, ce dernier place la dissection comme prélude indispensable à l'exercice de l'art. Il fait ainsi de la peinture un outil et une opportunité pour comprendre et travailler sur la mécanique humaine. Si, dans son œuvre, il dépasse l'application d'une anatomie systématique et statique pour privilégier le façonnage d'une attitude et d'un mouvement, ses études graphiques de chevaux révèlent les muscles d'un poitrail (pectoral descendant et transverse) ou d'une épaule (deltoïde) ${ }^{2}$, les tendons d'un membre antérieur (extenseur antérieur des phalanges, perforant, perforé, suspenseur du boulet) $)^{3}$, la corde du jarret d'un membre postérieur ${ }^{4}$, dans le but de répondre à un idéal physique, dans le respect de la notion de canon des proportions ${ }^{5}$. Dans l'esquisse du cheval cabré vu sur son profil gauche ${ }^{6}$, par exemple, il associe l'action des muscles principaux de l'encolure (splenius, trapèze (partie cervicale), stemo-céphalique et brachio-céphalique) aux contraintes subies par les vertèbres cervicales (atlas et axis) et résout ainsi l'équation de l'adaptation du geste à l'anatomie. Le dynamisme du mouvement se retrouve notamment dans la succession de la figuration du membre antérieur gauche et du postérieur droit, des différentes torsions de l'encolure et de la tête (dans au moins trois positions différentes).

Suivant le courant humaniste du XVI ${ }^{\mathrm{e}}$ siècle, les principes et les fondements de l'art sont théorisés par l'écrit. Dans ce contexte, l'idéal réaliste de la peinture du Nord fait concurrence à l'idéalisme italien. On peut ainsi faire le parallèle entre la description du cheval parfait du peintre flamand Karl Van Mander (1548-1606) et celle de l'écuyer Pierre de La Noue (15 ??-16 ??), dans laquelle la notion de proportions est associée à une 
relation au sensible et à la réalité de la beauté du vivant, qui détient une place fondamentale (tableau 1).

Tableau 1. La description du cheval parfait selon le peintre flamand Karl Van Mander et l'écuyer Pierre de La Noue

\begin{tabular}{|c|c|}
\hline Karl Van Mander, 1604 (trad. Noldus 2009) & Pierre de La Noue, 1620 \\
\hline $\begin{array}{l}\text { par trois cercles de dimensions déterminées, dont } \\
\text { l'un est la croupe, l'autre la poitrine, le troisième va } \\
\text { servir pour le corps; et de construire ensuite selon la } \\
\text { juste mesure la courbe du cou, et de faire tout sans } \\
\text { contradiction, dans les proportions. Vouloir une } \\
\text { chose est léger comme une plume, mais pouvoir le } \\
\text { faire est lourd comme du plomb }\end{array}$ & $\begin{array}{l}\text { apres en avoir propotionné le corps à ses } \\
\text { pieds, \& la teste \& le col, à l'une \& à l'autre } \\
\text { des ces parties, ils sont venus à } \\
\text { particulariser sur tout ce qui fait au merite } \\
\text { de chacune en particulier }\end{array}$ \\
\hline la belle stature & $\begin{array}{l}\text { le garot haut \& droit, \& si bien dilaté qu'on } \\
\text { puisse aysement voir la separation des } \\
\text { épaules }\end{array}$ \\
\hline les sabots sombres & la corne seiche, noire, lissée, creuse \& rond \\
\hline les jarrets courts ni trop courbes ni trop étroits & $\begin{array}{l}\text { ils luy ont donné les iarets \& faux du cerf, } \\
\text { pour plus prestement courir }\end{array}$ \\
\hline la poitrine large et grasse, ainsi que les épaules & $\begin{array}{l}\text { la poitrine enflée comme le iabot d'un } \\
\text { pigeon, \& large comme la iube ou le devant } \\
\text { d'un lyon, pour monstrer l'assistance de la } \\
\text { chaleur \& la vivacité de ses esprits }\end{array}$ \\
\hline les flancs ronds, le ventre court & $\begin{array}{l}\text { l'esquine courte \& ronde pour luy faire } \\
\text { mieux unir ses forces \& se comporter plus } \\
\text { gaillardement en ses actions \& mouvemens } \\
: \text { le boyau gros \& rond, \& proprement logé } \\
\text { fous les costes, à fin d'en cuire \& digerer } \\
\text { mieux ses alimens, \& d'en estre plus aisé à } \\
\text { retenir sous la felle }\end{array}$ \\
\hline le cou large et long & $\begin{array}{l}\text { ils luy ont formé le col long, déchargé, grélé } \\
\text { pres de la teste \& debandé pour la luy mieux } \\
\text { ramener }\end{array}$ \\
\hline une longue crinière & $\begin{array}{l}\text { ils ont embelly de crins longs, crespez \& } \\
\text { éparpillez, pour un asseuré témoignage de } \\
\text { force, de vigueur \& de bon temperament }\end{array}$ \\
\hline $\begin{array}{l}\text { la queue pendant jusqu'à terre - mais on peut la } \\
\text { représenter aussi bien relevée }\end{array}$ & $\begin{array}{l}\text { le tronc de la queüe long \& sec, \& bien } \\
\text { fourny de poil, pour marque de nerf \& de } \\
\text { bonne esquine }\end{array}$ \\
\hline les fesses rondes doivent trembler de graisse & la crouppe ronde \& cavë par le milieu \\
\hline
\end{tabular}




\begin{tabular}{|l|l|}
\hline la tête petite et maigre, sèche & $\begin{array}{l}\text { la teste, ils l'ont décharnée pour la } \\
\text { décharger comme celle du mouton, pour } \\
\text { mieux representer l'absence de l'abondance } \\
\text { du flegme }\end{array}$ \\
\hline le front osseux & $\begin{array}{l}\text { le front large \& tenant du cercle pour } \\
\text { marque d'une humeur superbe \& furieuse }\end{array}$ \\
\hline les yeux grands et saillants & $\begin{array}{l}\text { deux gros yeux noirs \& brillans, comme } \\
\text { ceux du boeuf \& du loup, pour découvrir la } \\
\text { syncerité deson ame \& la generosité de son } \\
\text { courage }\end{array}$ \\
\hline
\end{tabular}

On observe dans ces extraits issus de traités contemporains mais d'auteurs porteurs d'un savoir différent, celui de l'artiste qui représente le mouvement du cheval et celui de l'écuyer qui demande le mouvement au cheval, des similitudes troublantes qui existent dans la description des différentes parties du corps du cheval (taille, sabot, jarret, poitrail, flanc, encolure, crinière, queue, croupe, tête). Van Mander 2009 ; La Noue 1620

7 On trouve également chez ces deux auteurs des correspondances quant aux choix des robes (bai, alezan, blanc, noir) liés aux humeurs et aux tempéraments (air, feu, eau, terre) (tableau 2). Les artistes trouvent dans les manèges et dans le rôle des écuyers les exemples nécessaires à leur travail de figuration.

Tableau 2. La description des robes selon le peintre flamand Karl Van Mander et l'écuyer Pierre de La Noue

\begin{tabular}{|ll|l|}
\hline $\begin{array}{llr}\text { Karl Van Mander, } \\
1604 \quad \text { (trad. Noldus } \\
2009)\end{array}$ & Pierre de La Noue, 1620 \\
\hline $\begin{array}{l}\text { Les bais, les gris sont } \\
\text { les plus beaux }\end{array}$ & $\begin{array}{l}\text { le Bay chatain surpasse tous les autres en perfection \& bonté [...] } \\
\text { Le Bay clair est naturellement adroit \& sauteur [...] } \\
\text { Le gris blanc est accompagne de sang \& de flegme, \& doüé de grande } \\
\text { vigueur \& santé, \& arrive communément au poinct de la perfection par la } \\
\text { facilité \& franchise }\end{array}$ \\
\hline $\begin{array}{l}\text { les blancs et les } \\
\text { louvets les plus laids }\end{array}$ & $\begin{array}{l}\text { Le Blanc, vray portrait de l'eau, de son humidité \& de sa froideur, est } \\
\text { d'humeur l'ache \& foible [...] }\end{array}$ \\
Le gris louvet est de son temperament melancholique \& pesant
\end{tabular}

On observe dans ces extraits issus des mêmes traités que précédemment une concordance quant à la description des robes. La différence se fait au niveau de leur fonctionnalité : l'artiste y associe des qualités esthétiques, l'écuyer des qualités techniques.

Van Mander 2009 ; La Noue 1620

Daniel Roche note que Léonard de Vinci en fait écho dans son Traité de la peinture et dans les dessins préparatoires de la Bataille d'Anghiari ${ }^{7}$ (Roche 2015 : 370). Jan Van der Straet illustre l'académisme naissant avec ses planches sur les moments et les différentes strates de la société équestre: la course des chevaux en liberté, le duel 
équin, le cheval royal, le cheval libre et mal débourré, etc. Les chevaux y sont représentatifs d'une science écuyère et d'une société hérö̈que.

Fig.2. Étude pour la construction d'une statue équestre

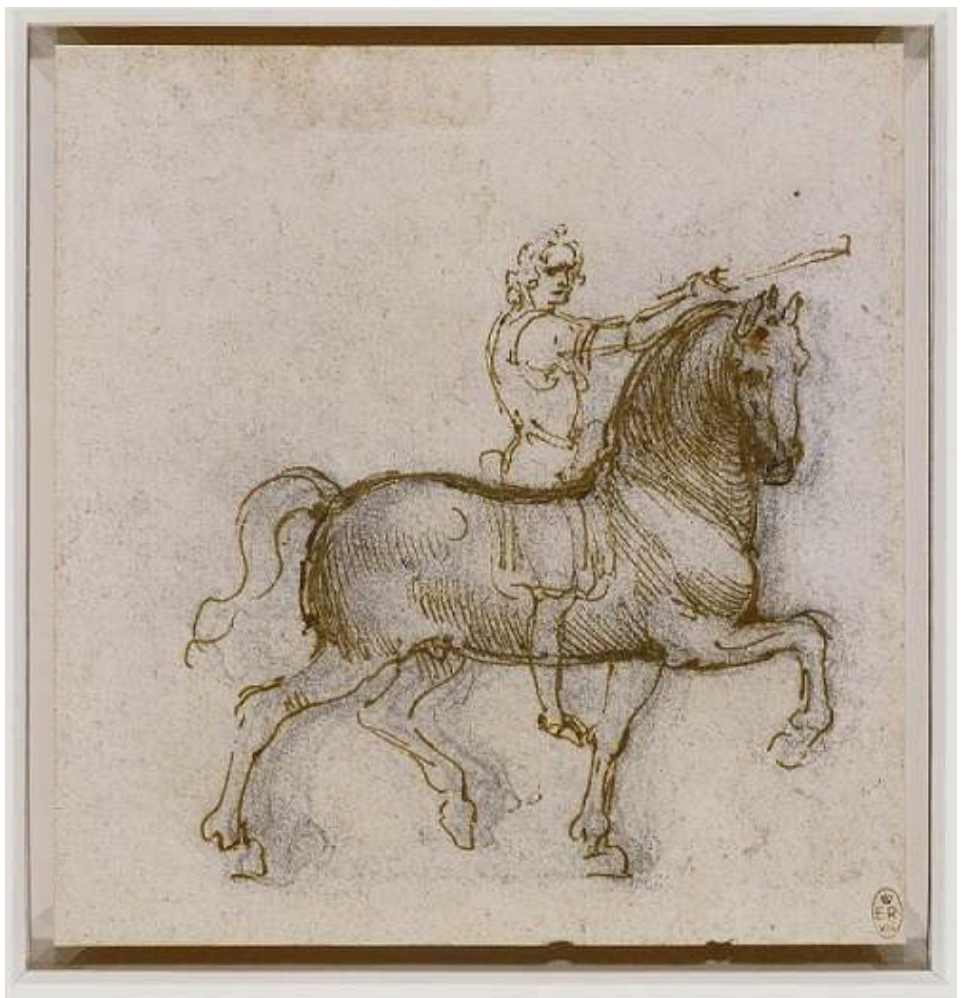

Dans cette étude graphique, Léonard de Vinci met en scène un cavalier sur un cheval au trot ou au passage, témoignage de la réalité de la race équine napolitaine de la fin du XVe siècle et du début du XVle siècle.

"A study for an equestrian monument c.1517-18. Pen and ink over black chalk | 15.4 x 14.5 cm (sheet of paper) | RCIN 912343 », Royal Collection Trust / (c) Her Majesty Queen Elizabeth II 2021

\section{Un modèle idéal}

La figure équestre traduit la construction des identités nationales de l'Europe de la Renaissance et des débuts des temps modernes. Elle témoigne d'une volonté de représenter "la réalité équine contemporaine, que ce soit par le biais de modèles caractérisés et de types de chevaux identifiables, ou encore de détails choisis de morphologie ou de physiologie équines, incluant les traces de certaines pratiques hippologiques et hippiatriques » (Fémelat $2015: 2$ ). Ainsi, à la fin du XVe siècle, on voit émerger le concept de race équine dans les élevages italiens des familles d'Aragon à Naples, Gonzague à Mantoue et Este à Ferrare. On retrouve dans les études préparatoires de Léonard de Vinci pour la construction de statues équestres ${ }^{8}$, qui reprennent systématiquement la figure d'un cheval au trot ou au passage reposant sur un bipède diagonal (un membre postérieur et le membre antérieur opposé), le morphotype du cheval napolitain: taille importante, poitrail et croupe éclatés, encolure en col de cygne rouée, chanfrein convexe. Ses qualités esthétiques et ses aptitudes naturelles sont alors recherchées dans le cadre de l'apparition des académies équestres au XVI ${ }^{\mathrm{e}}$ siècle. Salomon de La Broue (1552-vers 1610) dit de lui qu'il «sera 
nerveux, patient \& obeissant aux Chastiemens, bon a la main, alegre, dispost, de bonne mémoire, aise à raffermir de teste, propre à plusieurs sortes d'airs \& de maneges » (La Broue $1610: 9$ ). René Menou de Charnizay (1578-1651) le décrit comme étant "communément de plus forte taille que celuy d'Espagne, \& un peu plus chargé de chair, [il] ne differe en rien de toutes ses perfections, \& mesmes en ayant davantage: En ce qu'outre qu'il est capable de tout ce que peut faire l'autre, il est plus propre à travailler, \& ne s'use pas tant ny si-tost les jambes » (Menou de Charnizay 1651: 4). Enfin, William Cavendish (1593-1676) le tient en grande estime parce que " ce sont des galands chevaux; ceux [qu'il a vu] étoyent de grosse taille, toutefois de grande force, \& avoyent beaucoup d'espris » (Cavendish $1658: 15$ ).

10 En France, Antoine de Pluvinel (1552-1620) fait le constat suivant au début du XVII siècle :

\begin{abstract}
«Sire, plusieurs Provinces nous donnent des chevaux : ceux que nous avons le plus communément, viennent d'Italie, ou la plus part des races à present sont perduës \& abastardies [...] D'Espagne nous en avons rarement, encor ceux qui nous passent, ne sont pas les meilleurs. De Turquie, il nous en vient si peu que nous n'en devons pas faire cas, quoy qu'ils soient tres excellens, \& plus que ceux que i'ay nommez. Les Barbes nous sont plus communs, ordinairement bons, \& tous propres à faire quelque chose. L'Alemagne, la Flandre, \& l'Angleterre nous en donnent aussi, mais pour moy, ie trouve (Sire,) que ceux qui naissent en vostre Royaume sont aussi bons, ou meilleurs, qu'aucuns de ceux qui nous viennent de toutes ces nations estrangeres : car i en ay veu de Gascogne, d'Auvergne, de Limosin, de Poictou, de Normandie, de Bretagne, \& de Bourgogne de tres excellents [...] i'ay remarqué que ceux qui y naissent ont toutes les excellentes qualitez requises au beau \& bon cheval. Et pour moy, ie ne m'enquiers point de quel pays ils soient, quand ie les voy avoir bonne taille, beaux pieds, \& belles jambes, avec de la force, de la legereté, \& d'une bonne \& douce nature ; neantmoins ie fais grand estat des Barbes pour la Carriere, \& pour la grande inclination qu'ils ont à bien manier avec une dextérité, \& une grace plus particuliere que les autres » (Pluvinel $1627: 20-21$ ).
\end{abstract}

11 La seule planche de son traité réalisée par Crispin de Pas (1564-1637), dans laquelle le cheval est personnifié, est la figure d'un «Barbe bay [...] le parangon certes de tous les chevaux de Maneige du monde, tant pour sa beauté, que pour son excellence, à manier parfaictement, \& de bonne grace, terre à terre, \& à courbettes, avec tant de justesse, \& d'agilité, que ce n'est pas sans cause qu'il s'appelle le Bonite » (Pluvinel $1627: 20,21$ ).

Dans ce contexte, il faut attendre la fin du XVII siècle et l'intervention de l'État monarchique pour assister à la création des haras royaux. Colbert s'associe à Gabriel Calloët de Kerbrat et à François Alexandre Garsault pour constituer des normes qui définissent les beaux et bons chevaux selon les choix sociaux de l'époque. La politique d'élevage et les croisements "orientalisant» avec des chevaux barbes et turcs témoignent alors d'une réelle politique étrangère. William Cavendish recommande les croisements suivants : 
«Pour vôtre étalon, il n'y a vrayement aucun cheval meilleur qu'un beau Barbe bien choisy, ou qu'un beau cheval d'Espagne bien fait, \& bien marqué, affin de donner bonne teinture à vôtre haras [...] les Barbes \& les chevaux d'Espagne sont les meilleurs pour engendrer. Quant aux cavalles, je voudrois que vous choisisiez des belles cavalles d'Espagne pour faire race, ou quelques unes du Royaume de Naples, qui soient bien faites. Mais si vous ne pouves avoir aisement de celles-cy, choisissés des belles cavalles Angloises, lesquelles seront aussy bonnes qu'aucunes, pourveu qu'elles soyent de bon poil \& bien marquées; ce qui est aussy requis pour donner bonne teinture à vôtre haras, comme est le bon poil de l'étalon» (Cavendish 1658 : 20-21).

Fig.3. "Superbe Cheval de Spanie »

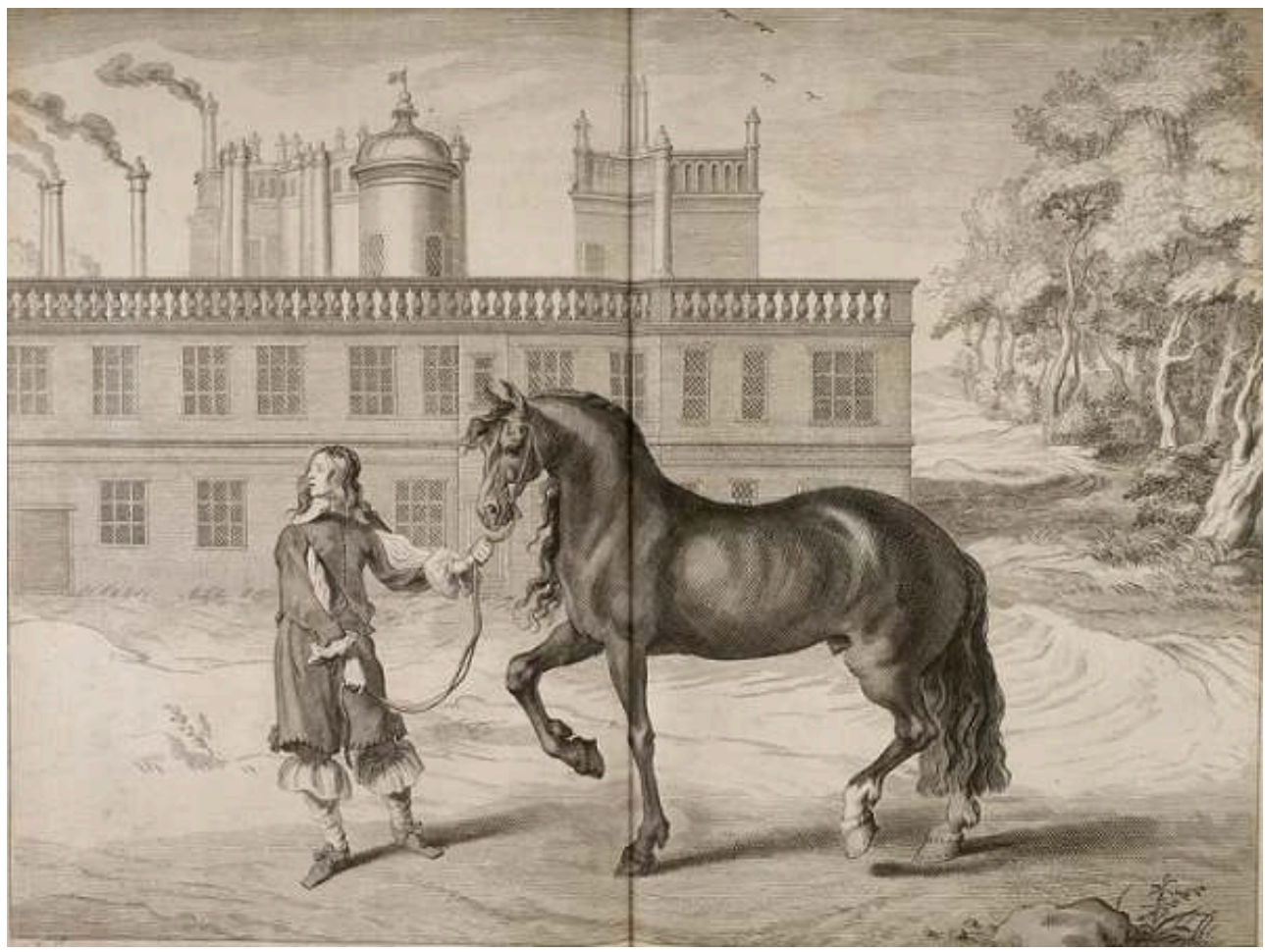

Planche d'Abraham von Diepenbeke illustrant un page tenant en main le «Superbe Cheval de Spanie » devant le château de Welbeck. On retrouve très distinctement les caractéristiques physiques de sa race : corps compact, encolure massive et bien portée, lonque crinière ondulée, allure relevée avec abaissement des hanches.

Cavendish 1658

On retrouve ainsi dans les planches de son traité réalisées par Abraham von Diepenbeke (1607-1675) le «Superbe Cheval de Spanie », « Makomilia, un Turke » et le « Nobilissimo Coursier Nappolitain ». 
Fig.4. « Makomilia, un Turke »

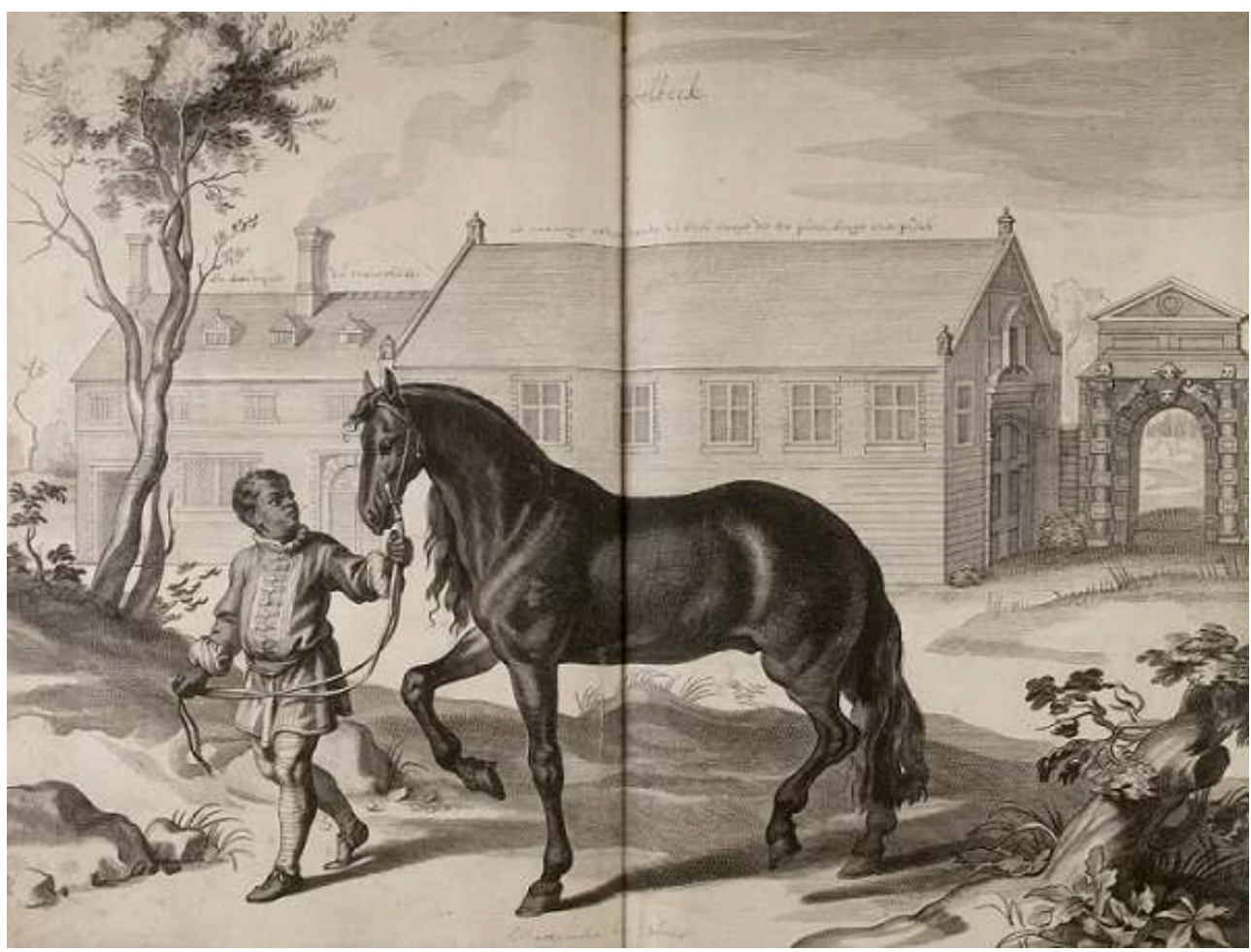

Planche d'Abraham von Diepenbeke illustrant un page tenant en main le cheval « Makomilia, un Turke » devant le château de Welbeck. On retrouve très distinctement les caractéristiques physiques de sa race : corps sec, fin et plat, encolure légère et arquée, hauteur du garrot sensiblement égale à la hauteur du corps.

Cavendish 1658

\section{Un modèle politique}

L'analyse et la compréhension du travail des artistes peintre et des sculpteurs en tant que vecteur entre le monde réel et l'imaginaire de son public est nécessaire pour interroger les choix de représentation fait par les illustrateurs des traités techniques équestres. Il est alors légitime de se demander si ces artistes qui doivent transmettre le visuel d'une pratique ont transformé le langage des canons artistiques du moment au service d'un énoncé technique et d'un projet pour favoriser la transmission d'un savoir. Il est également impératif de tenir compte de la fonction esthétique de ces productions dans leur rapport à la société. En effet, les canons artistiques peuvent imposer ou prohiber la plus réaliste des représentations de façon à répondre aux attentes de l'imaginaire du public. Dans ce cas, le contenu devient la forme. On peut alors rapprocher la représentation équestre d'une construction narrative et formelle de l'œuvre littéraire faisant intervenir les acteurs que sont la situation sociale, l'esprit d'invention d'une époque et l'imaginaire esthétique recherché par l'artiste. Les chevaux, essence de l'inspiration de l'art aux artistes, deviennent le moyen d'expression de différents et nombreux dynamismes sociaux, politiques et culturels, un reflet du monde nécessaire à sa compréhension. Ainsi, dans les études de Léonard de Vinci ${ }^{9}$, le détail accordé aux organes génitaux mâles exprime une dimension martiale du courage au combat (fig.5). Ils deviennent de fait un attribut du pouvoir. 
Fig.5. Étude du détail de l'arrière-main

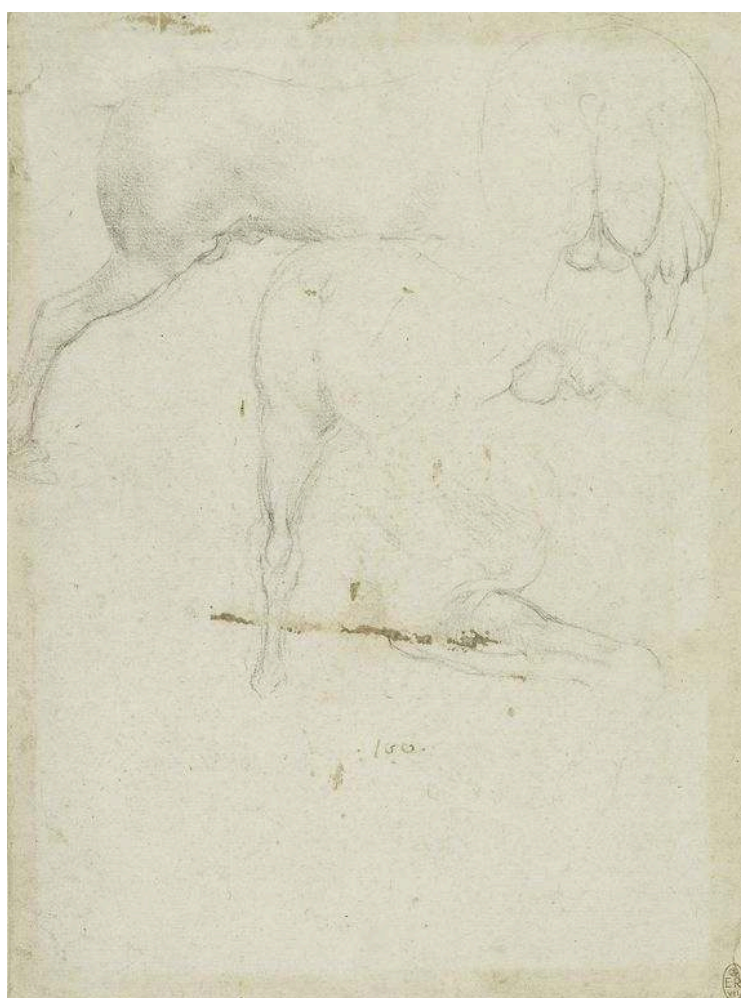

Dans cette étude graphique, Léonard de Vinci propose différentes vues (profil, dos) d'une arrière-main en accordant un soin tout particulier à la représentation des testicules et du fourreau.

"The hindquarters of horses c.1517-18. Black chalk | 22.8 × $16.8 \mathrm{~cm}$ (sheet of paper) | RCIN 912314 ", Royal Collection Trust / @ Her Majesty Queen Elizabeth II 2021

Le même souci du détail se retrouve dans la planche du Bonite, présenté en main par un page (Pluvinel $1627: 23$; fig.9), cheval offert par Monsieur le Grand au roi Louis XIII, ou bien encore sur celle figurant le roi à cheval en train de «garder sa bonne posture, \& executer à cheval de bonne grace tout ce qui se peut faire » (Pluvinel 1627 : 78 ; fig.6). 
Fig.6. Planche de Crispin de Pas représentant le roi à cheval

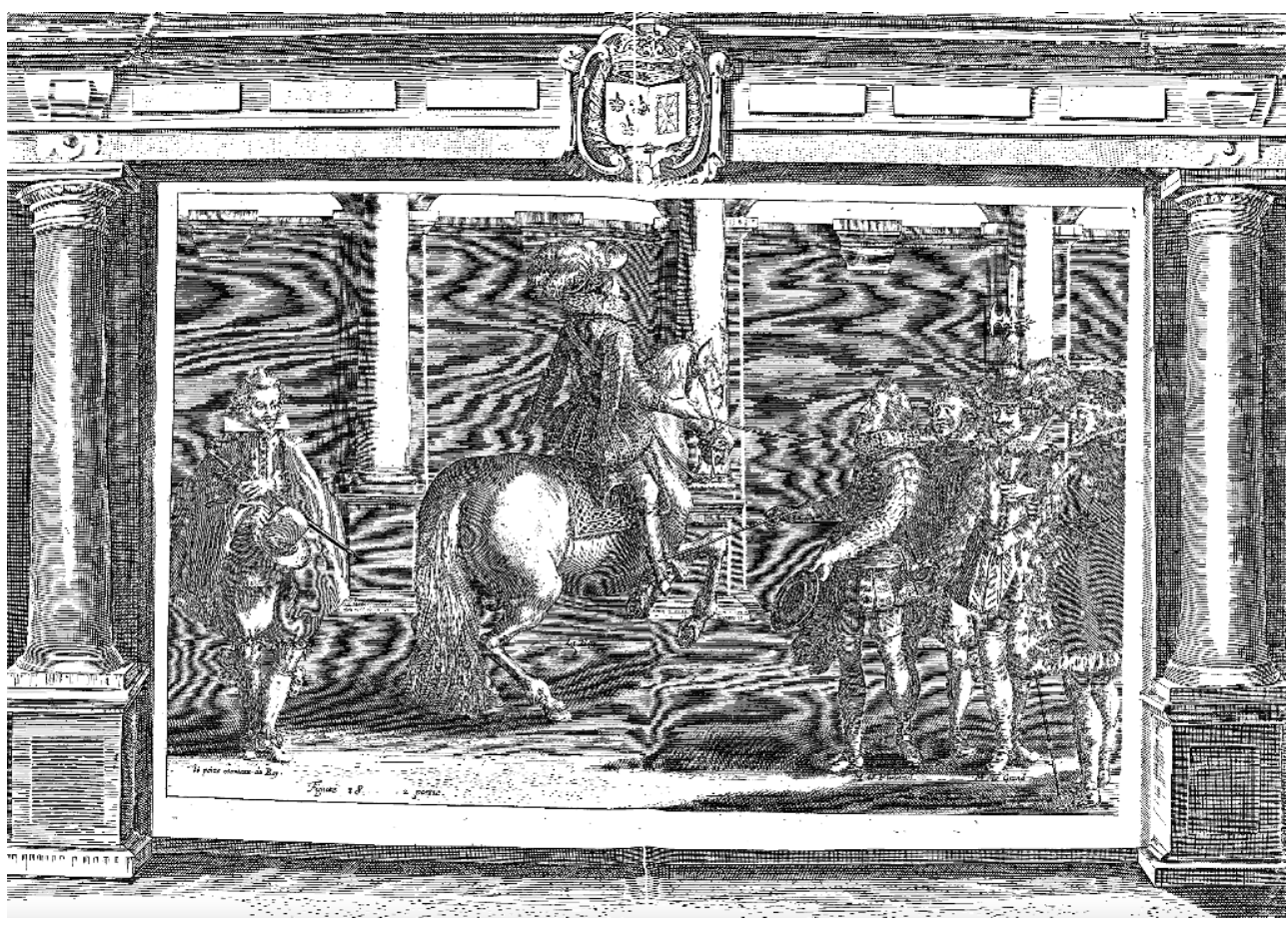

Pluvinel $1627: 78$

On retrouve dans les statues équestres de Léonard de Vinci un instrument de communication politique dans lequel le cheval glorifie son cavalier à la fois pour le commanditaire mais également pour son auteur. Ainsi, avec le projet monumental d'une statue équestre en hommage à François Sforza (1401-1466), fondateur de la maison Sforza, commandé par Ludovic Sforza (1452-1508), Duc de Milan, il veut supplanter l'œuvre d'Andrea del Verrocchio (1435-1488) dédiée à Bartolomeo Colleoni et celle de Donatello di Niccolò di Betto Bardi (1386-1466) dédiée à Gattamelata.

Dans ce type d'étude, la représentation du geste équestre est «l'expression artistique de la vision combinée d'un créateur et d'un commanditaire, conçue en fonction d'un public prédéterminé » (Fémelat $2015: 24$ ). La lecture peut se faire à deux niveaux qui sont indissociablement liés : celui du geste de l'artiste et celui du geste équestre. Ceuxci s'inscrivent dans la culture technique de l'époque durant laquelle ils ont été produits. Cette mise en scène de la société cavalière se révèle dans la représentation du cercle pédagogique présente dans les planches du traité d'Antoine de Pluvinel où le roi courre la bague, rompt en lice et combat à l'épée devant les grands du royaume (Pluvinel 1627, passim, fig.7). Le discours figuratif illustre alors les premières années du règne de Louis XIII et son appropriation de la raison cavalière. 
Fig.7. Planche de Crispin de Pas représentant le roi à cheval en train de courir la bague devant les grands du royaume

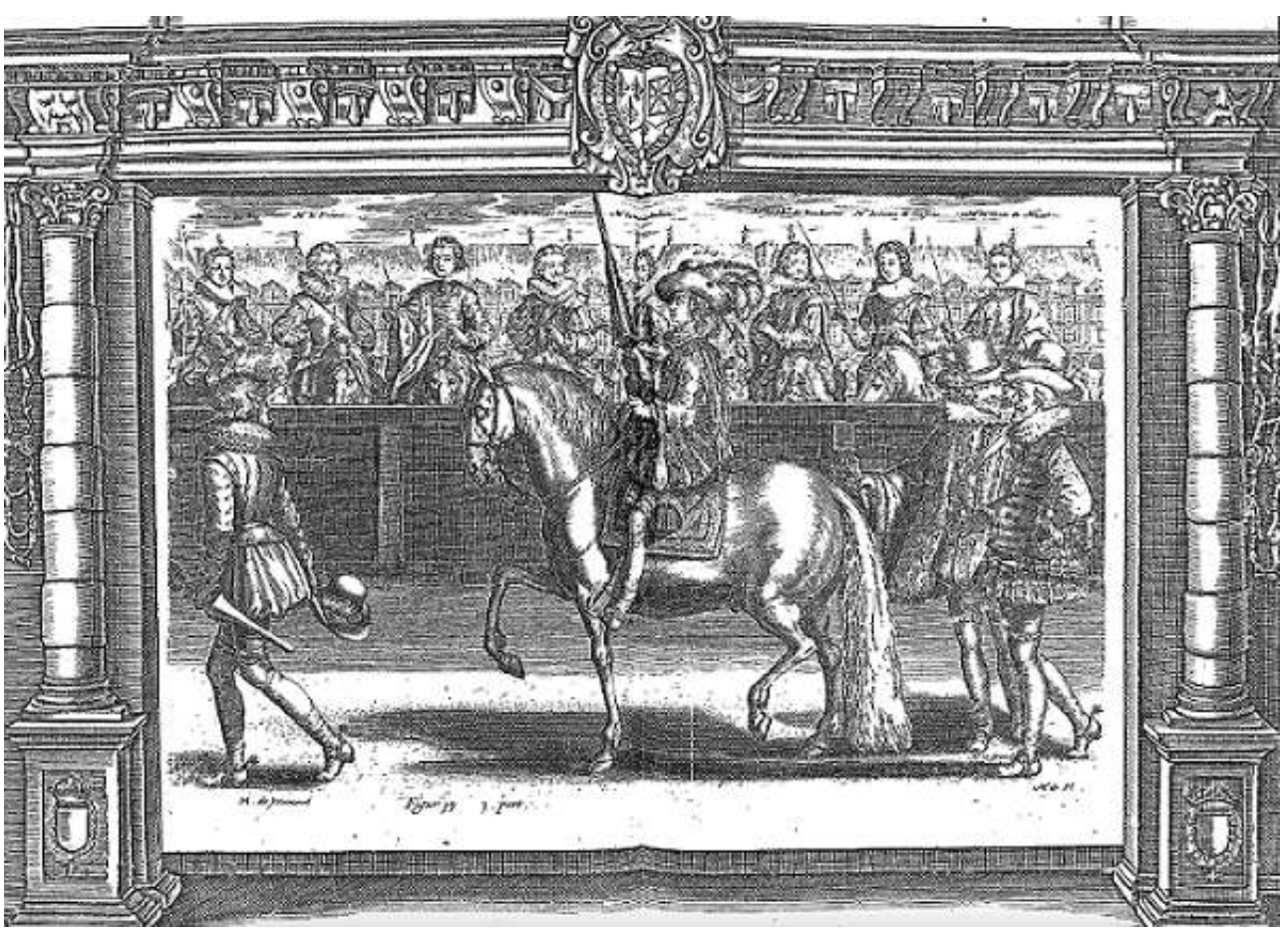

Pluvinel $1627: 154$

\section{Conclusion}

Le regard scientifique accordé par Léonard de Vinci à l'anatomie du cheval dans ses études d'après un modèle vivant devient un outil technique au service d'une idéalisation artistique du mouvement équestre. Du dynamisme de l'attitude du cheval découle la position du cavalier. La tension liée à la représentation de l'éphémère du geste vient de cette dualité des mouvements. Elle se retrouve dans les planches des traités techniques d'équitation des débuts des temps modernes. Dans l'idée d'un réalisme à la recherche d'une esthétique, le posé d'un membre illustre la cadence de l'allure. La dilatation des naseaux traduit quant à elle la vigueur de l'effort. L'expressivité des oreilles figure la confiance et l'attention du cheval envers la demande de son cavalier. Les plis de peau de l'angle tête encolure incarnent la pulsion du mouvement. Néanmoins, la représentation du cavalier reste figée dans l'instantané du mouvement.

19 Cette étude qui introduit une connexion entre art et science permet également d'apporter un regard nouveau sur la lecture des traités techniques, dans lesquels certains tentent de pallier le problème de la représentation du geste en naviguant entre réalisme et géométrisation du mouvement. Le cheval et l'homme sont désormais représentés de la manière la plus réelle possible, tandis qu'un tracé géométrique est introduit pour traduire le mouvement. On retrouve dans cette approche la recherche faite par Léonard de Vinci pour rationaliser le cheval idéal à travers ses études des proportions du corps équin annotées de commentaires et de mesures ${ }^{10}$. Enfin, il y a ceux qui font le choix de faire disparaître la figure du cheval et du cavalier au profit 
d'une schématisation maximum du mouvement, de façon à en simplifier la compréhension.

Cette mise en forme géométrique du geste technique n'est pas sans rappeler ce que Galilée écrit dans son pamphlet en réponse au père Grassi, au sujet d'une nature écrite en langage mathématique. L'objet mathématique devient une composante du langage, une plateforme jouant le rôle d'interface entre la nature du corps et l'Homme. À la différence de ce qui a été reproché à Alberti, à savoir le fait « d'avoir enfermé la beauté dans le carcan d'un modèle mathématique » (Boriaud 2007), la mise en image du geste technique dans le cadre de la réduction en art amène petit à petit à une rigueur du travail de l'artiste pour transmettre le savoir juste. Par le biais de figures et de calculs simples, il s'agit de proposer une modélisation du geste technique pour en favoriser sa transmission.

En ce sens, l'iconographie de la réduction en art (Dubourg-Glatigny, Vérin 2008) suit les principes artistiques venus de la Renaissance italienne, avec l'intégration de la perspective, des mathématiques et de la géométrie, dont les études équines de Léonard de Vinci sont une traduction ${ }^{11}$. La difficulté consiste à retrouver une vision tridimensionnelle, permettant l'adéquation entre le geste souhaité et sa représentation. Cela ramène à la question de la construction des lignes géométriques, notamment dans la controverse des points de fuite et de convergence évoquée par Jean-Pierre Le Goff, amenant à la perspective, dont le but est de donner une représentation la plus illusionniste qui soit d'un réel tridimensionnel (Le Goff 2007). Cette association entre art et mathématiques est le fruit des traités de deux peintres, Piero della Francesca (1412-1420) à Venise, et Albrecht Dürer (1471-1528), à Nuremberg. En plus de représenter sur une surface plane un mouvement qui se passe dans un milieu en trois dimensions, il s'agit pour l'artiste ou dans le cas qui nous intéresse, l'illustrateur du traité technique d'équitation, d'inscrire son œuvre dans un processus de pensée de l'espace mettant en scène un habitus visuel. On a dès lors une spatialisation de la représentation du mouvement associée à sa géométrisation.

\section{BIBLIOGRAPHIE}

BARBIN Evelyne, « Avant-propos », dans BARBIN Evelyne (dir.), Arts et sciences à la Renaissance, Paris, Ellipses, 2007, p.3-5

BORIAUD Jean-Yves, « Alberti et la technique de l'imitation », dans BARBIN Evelyne (dir.), Arts et sciences à la Renaissance, Paris, Ellipses, 2007, p.145-155

CAVENDISH William, La Methode Nouvelle \& Invention extraordinaire de dresser les Chevaux, les travailler selon la nature par la subtilité de l'art ; laquelle n'a jamis été treuvée que Par le tres-noble, haut et tres-puissant Prince Guillaume, Marquis et Comte de Newcastle, Vicomte de Mansfield, Baron de Bolsover et Ogle Seigneur de Cenvendish. Bothel et Hepwel; Pair d'Angleterre Qui eut le charge et l'honneur d'estre Gouverneur du Serenissime Prince de Galles en sa jeunesse et maintenant Roy de la Grande Bretagne; Et d'avantage qui est Lieutenant pour le Roy de la Comté de Nottingham et la Forest de Sherwood; Captaine general en toutes provinces outre la Rivière de Trent et autres endroits du Royaume d'Angleterre. Gentil- 
homme de la Chambre du Lit du Roy ; Conseiller d'Etat et Privé ; Et Chevalier de l'ordre tres-noble de la Iartiere etc. Traduit de l'Anglois de l'Auteur en François par son Commandement, Anvers, Jacques Van Meurs, 1658

CHAUVIRÉ Christiane, L'Essayeur de Galilée (introduction, traduction et commentaire), Annales littéraires de l'Université de Besançon, Paris, Les Belles Lettres, 1980

DUBOURG GLATIGNY Pascal, VÉRIN Hélène, Réduire en art. La technologie de la Renaissance aux Lumières, Paris, Éditions de la Maison des Sciences de l'Homme, 2008

FÉMELAT Armelle, « Des chevaux réels et un cheval idéal : naturalisme et idéalisation des chevaux des portraits équestres italiens des Trecento et Quattrocento », In Situ, n²7 ; 2015

GARIN Eugenio (dir.), L'homme de la Renaissance, Paris, Points, 2002

KELLER André, Le traité de peinture de Léonard de Vinci, Paris, Éd. Jean de Bonnot, 1977

LA BROUE Salomon (de), Le Cavalerice François. Composé par Salomon de La Broue, Escuyer de l'Escuirie du Roy et de Monseigneur le Duc d'Espernon, Contenant les Preceptes principaux quil faut observer, exactement pour bien dresser les Chevaux aux exercices de la carriere et de la campagne. Le tout divise en trois livres. Le premier traicte de l'ordre general et plus facile des susdits exercices et de la propreté du Cavalier, Le second des modernes et plus justes proportions de tous les plus beaux airs et maneges, Le troisiesme des qualitez de toutes les parties de la bouche du Cheval et des divers effets de plusieurs brides differentes pourtraites et representees par leurs justes mesures aux lieux necessaires. Troisieme Edition reveue et augmentee de beaucoup de leçons et figures par l'Autheur, Paris, Abel l'Angelier au premier pilier de la grand Salle du Palais, 1610

LA NOUE Pierre (de), La Cavalerie Françoise et Italienne ou L'art de bien dresser les chevaux, Strasbourg, Iac de Heyden, 1620

LE GOFF Jean-Pierre, « De points de distance en points de fuite, une histoire non linéaire de la perspective ? ", dans BARBIN Evelyne (dir.), Arts et sciences à la Renaissance, Paris, Ellipses, 2007, p. $33-67$

LE NEN Dominique, «Léonard de Vinci et sa vision de la main », dans BARBIN Evelyne (dir.), Arts et sciences à la Renaissance, Paris, Ellipses, 2007, p.11-31

MENOU DE CHARNIZAY René, La pratique du cavalier ou l'exercice de monter a cheval. Qui enseigne la methode de reduire les Chevaux dans l'obeïssance des plus beaux Airs \& Maneiges. Par Messire Rene de Menou, Chevalier, Seigneur de Charnizay. Reveu, corrige et augmente par luy mesme; Avec les Figures, pour en donner l'intelligence. Ensemble un traite des moyens d'empescher les Duels, \& bannir les Vices qui les causent, Paris, Guillaume Loyson et Jean-Baptiste Loyson, 1651

MÉTIN Frédéric, « Images de la nature, images de l'homme dans les ouvrages de géométrie pratique ", dans BARBIN Évelyne (dir.), Arts et sciences à la Renaissance, Paris, Ellipses, 2007, p. 285-308

PLUVINEL Antoine (de), L'instruction dv Roy en l'exercice de monter a cheval. Par Messire Antoine de Pluvinel, son Soubs Gouverneur Conseiller, en son Conseil d'Estat, Chambellan ordinaire, \& son Escuyer principal. Lequel respondant a sa Maieste, lui faict Remarquer l'Excellence de sa Methode, pour reduire les chevaux en peu de temps à l'obeïssance des justes proportions de tous les plus beaux Airs \& Maneiges. Le tout enrichy de grandes figures en Taille douce, representant les vrayes \& naifves actions des hommes \& des Chevaux en tous les Airs, \& Maneiges, Courses de Bague, Rompre en lice au Quintan, \& combattre à l'Espée, Ensemble les figures des Brides, les plus necessaires à cet usage, Desseignees et Gravees par Crispian de Pasle Ieune, Paris, Pierre Rocolet, en la gallerie au Palais, aux Armes de la Ville, 1627 
ROCHE Daniel, La culture équestre de l'Occident XVI ${ }^{e}$-XIX ${ }^{e}$ siècle. L'ombre du cheval. Tome III. Connaissance et passion, Paris, Fayard, 2015

VAN DER STRAET Jan, Equile Ioannis Austriaci Caroli V. Imp. F. In quo omnis generis generosissimorum equo : rum ex varÿs orbis partibus insignis delectus. Ad vivum omnes desineati à celeberrimo pictore Iohanne Stradano Belga Brugensi. Et à Philippo Galleo editi, Anvers, Philippe Galle, s.d.

VAN MANDER Karel, Principe et fondement de l'art noble et libre de la peinture, trad. NOLDUS Jan Willem, Paris, Les Belles Lettres, 2009

VINCI Léonard (de), Les carnets, trad. MACCURDY, Paris, Gallimard, 2000

\section{NOTES}

1. L'exposition Leonardo da Vinci: A Life in Drawing s'est tenue à la Queen's Gallery de Buckingham Palace (Londres) entre mai et octobre 2019, à l'occasion du 500 anniversaire de la mort du maitre.

2. «Studies of a horse, c.1490. Metal point on blue-grey prepared paper $\mid 21.4$ x $16.0 \mathrm{~cm}$ (sheet of paper) | RCIN 912321 ", étude graphique conservée à Windsor Castle, Royal Library sur laquelle on peut voir le profil du corps entier d'un cheval avec différents positionnements pour les membres postérieurs et des lignes verticales indiquant les proportions des différentes parties du corps, le détail de face d'un poitrail auquel est rattaché le détail du membre antérieur droit et le croquis du membre antérieur gauche, le détail de trois quart face d'un poitrail auquel est rattaché le début du membre antérieur gauche.

3. "Recto: Horses' fore-legs, including one raised and bent. Verso: A sketch of Leonardo, the head of a youth, and a horse's legs, c.1517-18. Recto: Black chalk. Verso: Pen and ink, black chalk| 26.0 x $16.7 \mathrm{~cm}$ (sheet of paper) | RCIN 912300 ", étude graphique conservée à Windsor Castle, Royal Library sur laquelle on peut voir différentes vues d'un membre antérieur gauche (intérieur, face, extérieur) posé à la verticale et la vue de profil d'un membre antérieur droit replié en mouvement.

4. "Studies of a horse, c.1517-18. Black chalk, pen and ink, on paper washed buff $\mid 20.3 \times 15.8 \mathrm{~cm}$ (sheet of paper) | RCIN 912313 ", étude graphique conservée à Windsor Castle, Royal Library sur laquelle on peut voir différentes vues d'un membre postérieur gauche replié en mouvement (dos, face, profil), la vue de profil comprend l'intégralité de l'arrière main (croupe, postérieurs gauche et droit, queue).

5. «Le peintre qui a la connaissance de la nature des nerfs, muscles et tendons saura bien, dans le mouvement d'un membre, combien de tendons, et lesquels, provoquent ce mouvement, et quel muscle en se gonflant, est cause du raccourcissement du tendon. Il saura ainsi représenter les muscles, de façon diverse et universelle, par les effets variés des personnages, et il ne fera pas comme beaucoup qui, dans des gestes différents, font toujours ressortir les mêmes choses dans les bras, le dos, les poitrines et les jambes : ce chose-là, rajoute-t-il, ce ne sont pas de minces erreurs » (Keller $1977: 63)$.

6. «A rearing horse, c.1503-4. Red chalk, pen and ink | 15.3 x 14.2cm (sheet of paper) | RCIN 912336 ", étude graphique conservée à Windsor Castle, Royal Library.

7. « Recto: A horse and rider, and studies for Leda. Verso: Mortars bombarding a fortress, c.1503-4. Black chalk, pen and ink | 28.7 x 40.5cm (sheet of paper) | RCIN 912337 »; « Recto: A rearing horse, and heads of horses, a lion and a man. Verso: Notes and diagrams on astronomy and geometry, and the head of a horse, c.1503-4. Recto: Pen and ink, wash, a little red chalk. Verso: Pen and ink, traces of black chalk | 19.6 x 30.8cm (sheet of paper) | RCIN $912326 »$; « Recto: Cavalry skirmishes. Verso: Cavalry skirmishes, c.1503-4. Charcoal | 21.5 x 38.5cm (sheet of paper) |RCIN $912338 »$; «A cavalcade, c.1503-4. Black chalk | 16.0 x $19.7 \mathrm{~cm}$ (sheet of paper) | RCIN 912339 »; « Galloping and kicking horses, and a foot soldier, 
c.1503-4. Red chalk | 16.8 x 24.0cm (sheet of paper) | RCIN $912340 »$, études graphiques conservées à Windsor Castle, Royal Library.

8. «A study for an equestrian monument, c.1517-18. Black chalk $\mid 12.2 \times 17.6 \mathrm{~cm}$ (sheet of paper) | RCIN 912341 »; "A study for an equestrian monument, c.1517-18. Pen and ink over black chalk| $15.4 \mathrm{x}$ $14.5 \mathrm{~cm}$ (sheet of paper) |RCIN 912343 »; " Recto: A study for an equestrian monument. Verso: Scribbled lines (part of the hair and bust of a young lady in profile) c.1490. Pen and ink $\mid 2.8 \times 3.6 \mathrm{~cm}$ (sheet of paper) | RCIN 912345 »; « Designs for an equestrian monument, c.1517-18. Black chalk|27.8 X $18.4 \mathrm{~cm}$ (sheet of paper) | RCIN 912342 »; « Designs for an equestrian monument, c.1517-18. Black chalk, pen and ink | 20.3 x $14.3 \mathrm{~cm}$ (sheet of paper) | RCIN 912344 "; « Designs for an equestrian monument, c. 1517-18. Black chalk | 26.7 x 16.1cm (sheet of paper) |RCIN 912359 »; "Designs for an equestrian monument, c.1517-18. Black chalk, pen and ink | $22.4 \mathrm{x} 16.0 \mathrm{~cm}$ (sheet of paper) |RCIN 912360 »; "Recto: A study for the Trivulzio monument. Verso: A study for an equestrian monument. Recto: c. 1510-12 and c.1517-18. Verso: c.1517-18. Recto: Red chalk, pen and ink. Verso: Black chalk, gone over in part with pen and ink | $21.7 \times 16.9 \mathrm{~cm}$ (sheet of paper) | RCIN $912356 »$, études graphiques conservées à Windsor Castle, Royal Library.

9. "Horses' legs and hind-quarters, c.1478-80. Pen and ink and black chalk on orange prepared paper | 13.4 x $18.8 \mathrm{~cm}$ (sheet of paper) | RCIN 912306 »; " The hindquarters of horses, c.1517-18. Black chalk | $22.8 \times 16.8 \mathrm{~cm}$ (sheet of paper) | RCIN 912314 » études graphiques conservées à Windsor Castle, Royal Library sur lesquelles on peut voir notamment des membres postérieurs, qui révèlent le soin accordé à la représentation des testicules et du fourreau.

10. « Horses' heads and forelegs, with measurements, c.1490 and c.1508. Black chalk and pen and ink on pale pinkish-buff prepared paper | 27.5 x $19.6 \mathrm{~cm}$ (sheet of paper) | RCIN 912286 »; " A horse divided by lines, c.1490. Metal point on blue-grey prepared paper | 22.1 x $11.0 \mathrm{~cm}$ (sheet of paper) | RCIN $912290 »$; « Horses' forelegs divided by lines, c.1517-18. Pen and ink over traces of black chalk $\mid 25.7$ x $16.6 \mathrm{~cm}$ (sheet of paper) | RCIN 912293 »; "A horse's left foreleg, with measurements c.1490-92. Lead point, pen and ink, staining | 25.0 x $18.7 \mathrm{~cm}$ (sheet of paper) | RCIN $912294 »$; «A horse with lines of proportion, c.1480. Lead point, pen and ink | $29.8 \times 29.0 \mathrm{~cm}$ (sheet of paper) | RCIN 912318 "; " A horse in left profile, with measurements, c.1490.Metal point and pen and ink on blue-grey prepared paper, the outlines incised; sharply folded, the upper half damaged by damp | 32.4 x $23.7 \mathrm{~cm}$ (sheet of paper) | RCIN 912319 » études graphiques conservées à Windsor Castle, Royal Library.

11. "Ceux qui s'éprennent de là sans connaître la science ressemblent à des capitaines qui, sur des bateaux sans gouvernail ou sans boussole, ne sauraient jamais sur quoi ils mettent le cap. Il faut toujours bâtir la pratique sur un bon fondement théorique. La perspective en est le guide et la porte et, sans elle, on ne fait rien de bon. » (Keller $1977: 56$ ).

\section{RÉSUMÉS}

La place du cheval dans l'histoire des réalités figuratives est majeure. Et dans les traités d'équitation, sa représentation fait partie intégrante du discours. Pour autant, la représentation picturale du geste technique dans les traités équestres du début de la période moderne fait écho à la conception artistique de Léonard de Vinci avec une dimension scientifique, particulièrement présente dans ses études graphiques du cheval. Dans cet article, nous verrons d'abord comment l'iconographie des traités traduit en deux dimensions le mouvement instantané et la sensation de la monte, à l'instar de Léonard. Puis, en nous interrogeant sur la place du réalisme dans cette 
représentation des gestes du cheval et de son cavalier, nous verrons que cette vision d'une réalité idéalisée est bien au service d'un instrument politique.

The horse plays a great role in the history of figurative realities. And in equestrian treatises, its representation is an integral part of the discourse. However, in the early modern period, the pictorial representation of the technical gesture in equestrian treatises echoes Leonardo da Vinci's artistic conception with a scientific dimension, particularly present in his graphic studies of the horse. In this paper, we will first see how the iconography of the treatises translates the instantaneous movement and sensation of riding into two dimensions, following the example of Leonardo. Secondly, by examining the place of realism in this representation of the gestures of the horse and rider, we will see that this vision of an idealized reality is indeed at the service of a political instrument.

\section{INDEX}

Mots-clés : histoire des techniques, geste technique, dessin, conception, équitation, traité technique, cheval

Keywords : history of technology, technical gesture, drawing, design, equitation, technical treaty, horse

\section{AUTEUR}

\section{ANNE-SOPHIE RIETH}

Anne-Sophie Rieth est doctorante en histoire des techniques à l'université Paris 1 PanthéonSorbonne (Institut d'Histoire Moderne et Contemporaine/UMR 8066), sous la direction d'AnneFrançoise Garçon. Ses recherches portent sur l'histoire de la pensée technique équestre en France pendant l'époque moderne à partir de l'étude des traités de réduction en art. Il s'agit d'interroger la mise en récit du geste équestre à travers l'étude de la construction des énoncés techniques, en tant qu'articulation entre pensée technique et transmission du savoir technique, ainsi que sur la détermination du référent et de la légitimation de cette pensée. Une seconde approche porte sur la compréhension et l'interprétation du geste technique à partir de l'étude de sa représentation. Dans la lignée de ce dialogue entre pratique et théorie, elle a élaboré une fiche d'inventaire du patrimoine culturel immatériel de France sur la pratique des maréchaux-ferrants de la Garde Républicaine publiée sur le site du Ministère de la Culture. 